\title{
On transvaginal endoscopy for elucidating pathogenesis and treatment of ovarian endometrioma
}

\author{
Michelle Nisolle
}

Received: 3 January 2014 / Accepted: 15 January 2014 / Published online: 30 January 2014

(C) Springer-Verlag Berlin Heidelberg 2014

Ovarian endometriosis is largely discussed in the literature and still the pathogenesis and the surgical management remain a source of controversy. The original article by Sampson indicated that the site of perforation could represent the stigma of invagination and that perforation of the so-called chocolate cyst led to spillage, spread of peritoneal endometriosis and adhesions [1]. The findings of Hughesdon contradicted Sampson's hypothesis as he suggested that adhesions are not the consequence but rather the cause of endometrioma formation [2]. This theory of invagination of the ovarian cortex due to an accumulation of menstrual debris from the shedding and bleeding of active implants has been confirmed by Brosens et al. performing laparoscopic ovarioscopy and biopsies [3].

The performance of transvaginal endoscopy (TVE) lead to the possibility to observe small ovarian endometrioma. Gordts et al. demonstrate in a study published in this issue of Gynecologic Surgery that endometrioma of less than $10 \mathrm{~mm}$ could be formed on the ovarian surface even in absence of adhesions with the pelvic wall [4]. Their study is crucial as it provides, for the first time, evidence for both theories, the Sampson's one suggesting that adhesions are not a prerequisite for endometrioma formation as well as the observation of Hughesdon demonstrating the formation of endometrioma by invagination of the ovarian cortex.

The second point of discussion is what is the optimal surgical management for future fertility by maximum preservation of normal ovarian cortex which is the pool of normal ovocytes. Firstly, ovarian endometriosis per se has been shown to be associated with oxidative stress on surrounding normal ovarian tissue. It is potentially responsible for the decrease of the ovarian reserve [5]. In a recent study, Liu

M. Nisolle $(\bowtie)$

Department Obstetrics and Gynecology, University of Liege, Hôpital de la Citadelle, 4000 Liège, Belgium

e-mail: michelle.nisolle@chrcitadelle.be et al. demonstrated that reactive oxygen species levels in serum and follicular fluid were significantly higher in patients with endometriosis as compared to patients without endometriosis [6]. These findings indicate that surgery is mandatory to avoid the deleterious effect of ovarian endometriosis on the remaining normal ovarian tissue. Even if ovarian cystectomy is associated with a reduction of ovarian function, this technique, according to the recent ESHRE guidelines on endometriosis, is still considered as the most beneficial in terms of recurrence [7].

Nevertheless, ovarian cystectomy by stripping remains a complex surgical procedure, associated in some cases with heavy bleeding requiring extensive coagulation. The complexity is partly due to the development of a fibrotic capsula, typical for ovarian endometrioma, due to reaction by the host towards the presence of endometriotic tissue [8]. The second difficulty with performing complete cystectomy is related to the depth of the endometrioma, as the dissection must be continued as far as the ovarian hilus. To avoid such technical difficulties and risks of damaging normal ovarian tissue, the laser should also be used in order to selectively vaporize the cyst wall specially in the deepest part of the endometrioma.

It has been recently shown that ovarian endometriosis already develops during adolescence, usually as superficial lesions characterized by hypervascularization, at stage I or II in the majority of cases [9] The treatment of adolescent endometrioma conducted by TVE should be beneficial as it would protect against the oxidative stress on the normal ovarian cortex and it should associated with a decreased risk of premature ovarian failure.

In conclusion, as ovarian endometriosis is per se deleterious for the remaining ovarian tissue, and because its surgical management can be as well, we have to define the place of the TVE both in the diagnostic step as well as its early treatment, both in adolescent patients suffering from pelvic pain and women suffering from infertility. 
Conflict of interest Michelle Nisolle declares that she has no conflict of interest.

\section{References}

1. Sampson JA (1921) Perforating haemorrhagic (chocolate) cysts of the ovary. Arch Surg 3:245-323

2. Hughesdon PE (1957) The structure of endometrial cysts of the ovary. J Obst Gynaecol Br Emp 64(4):481-487

3. Brosens IA, Puttemans PJ, Deprest J (1994) The endoscopic localization of endometrial implants in the ovarian chocolate cyst. Fertil Steril 61(6): 1034-1038

4. Gordts S, Puttemans P, Gordts Sy, Valkenburg M, Brosens I, Campo R (2013) Transvaginal endoscopy and small ovarian endometriomas: unravelling the missing link? Gynecol Surg (in press)
5. Matsuzaki S, Schubert B (2010) Oxidative stress status in normal ovarian cortex surrounding ovarian endometriosis. Fertil Steril 93(7): 2431-2432

6. Liu F, He L, Liu Y, Shi Y, Du H (2013) The expression and role of oxidative stress markers in the serum and follicular fluid of patients with endometriosis. Clin Exp Obstet Gynecol 40(3): 372-376

7. Hart RJ, Hickey M, Maouris P, Buckett W (2008) Excisional surgery versus ablative surgery for ovarian endometriomata. Cochrane Database Syst Rev (2):CD004992

8. Alvarez-Gonzalez ML, Frankenne F, Galant C, Marbaix E, Foidart JM, Nisolle M, Beliard A (2009) Mixed origin of neovascularisation of human endometrial grafts in immunodeficient mouse models. Hum Reprod 24(9):2217-2224

9. Closon F, Brichant G, Tebache L, Pinzauti S, Nisolle M (2013) L'endométriose de l'adolescente. Mt Médecine de la Reproduction. Gynécol Endocrinol 15(3):228-233 